\title{
Current Trends in Implantable Left Ventricular Assist Devices
}

\author{
Jens Garbade, Hartmuth B. Bittner, Markus J. Barten, and Friedrich-Wilhelm Mohr \\ Department of Cardiac Surgery, Leipzig Heart Center, University of Leipzig, Struempellstraße 39, 04289 Leipzig, Germany
}

Correspondence should be addressed to Jens Garbade, garbade@med.uni-leipzig.de

Received 24 December 2010; Revised 1 March 2011; Accepted 1 March 2011

Academic Editor: H. O. Ventura

Copyright () 2011 Jens Garbade et al. This is an open access article distributed under the Creative Commons Attribution License, which permits unrestricted use, distribution, and reproduction in any medium, provided the original work is properly cited.

\begin{abstract}
The shortage of appropriate donor organs and the expanding pool of patients waiting for heart transplantation have led to growing interest in alternative strategies, particularly in mechanical circulatory support. Improved results and the increased applicability and durability with left ventricular assist devices (LVADs) have enhanced this treatment option available for end-stage heart failure patients. Moreover, outcome with newer pumps have evolved to destination therapy for such patients. Currently, results using nonpulsatile continuous flow pumps document the evolution in outcomes following destination therapy achieved subsequent to the landmark Randomized Evaluation of Mechanical Assistance for the Treatment of Congestive Heart Failure Trial (REMATCH), as well as the outcome of pulsatile designed second-generation LVADs. This review describes the currently available types of LVADs, their clinical use and outcomes, and focuses on the patient selection process.
\end{abstract}

\section{Introduction}

Heart transplantation is still the therapy of choice for patients with sustained heart failure resistant to any medical therapy. More than 16 million people are currently diagnosed with chronic heart failure (CHF) in Europe and the United States, where its prevalence averages $2.5 \%$ of the normal population $[1,2]$. CHF increases significantly after age 65 , and the population in this group will double within the next 20 years, suggesting heart failure incidence will similarly [3].

In the last decades, long waiting times for cardiac transplantation and subsequent increased mortality have led to an increase in the use of left ventricular assist devices (LVADs). Permanent mechanical circulatory support by new, smaller devices is a promising therapeutic option developed to provide an alternative to transplantation and to reduce mortality on the heart waiting list. The primary focus in this field was to develop a total artificial heart $(\mathrm{TAH})$, but this has had limited success and, as a result, shifted attention to ventricular assist devices. The firstgeneration of implantable ventricular assist devices (VADs) were pulsatile, volume-displacement pumps. The start of the modern LVAD era began with the introduction of the HeartMate XVE in 1998. Although the XVE launched amidst great fanfare and high expectations, the device failed to displace the long-standing view that mechanical ventricular support was merely an expensive gimmick. These devices provide excellent circulatory support and improve survival until heart transplantation. However, they have many application limitations, such as a large volume, the excessive surgical dissection required for placement of the device, presence of a large diameter driveline, noisy pump operation, and particularly limited mechanical durability. Other complications are bleeding, infections and thromboembolic events. During the succeeding decade, vast improvements in pump design resulted in a new crop of LVADs, whose attributes are transforming LVAD therapy into a kind of standard of care for end-stage heart failure [4-17]. LVAD therapy has now evolved into a solution which is strikingly superior to optimal medical therapy $[4-6,11]$.

The one factor most responsible for the advancement of LVAD therapy into the standard of care is the dramatic improvement in clinical outcomes seen in recent years $[4$, $12,17]$. Smaller nonpulsatile continuous-flow blood pumps (second- and third-generation LVADs) became available. Because of their simpler design (no mechanical bearings, no mechanical or biological valves), these devices showed a potentially longer durability $[9,12-14,18-22]$.

This paper describes the currently available types of LVADs, their clinical use and outcomes, patient selection, and further directions on this growing field. 


\section{Left Ventricular Assist Device in Practise and Outcome}

2.1. First Generation of LVAD Types. The most common and routinely used pulsatile devices are paracorporeal pumps such as Thoratec's paracorporeal ventricular assist devices (Figure 1, PVAD; Thoratec Inc.; Pleasanton, Calif, US) and the Berlin Heart Excor (Berlin Heart AG, Berlin, Germany), and implantable pumps such as HeartMate XVE (Figure 1, Thoratec Inc.), or Novacor (World Heart Corp., Oakland, Calif).

The HeartMate LVAD was first used in a clinical trial starting in 1986 as a pneumaticly actuated system that required a large cumbersome console that did not allow patients much mobility outside the hospital. This system proved to be effective as a long-term support device with the end goal of heart transplantation [23]. The HeartMate System underwent years of development and, in 1991, a clinical trial of a vented electric (VE) model was begun. This electric system allowed a greater amount of mobility with portable battery units carried in a holster [24]. Since then, both models have shown a $60 \%$ to $70 \%$ rate of survival to transplantation. The worldwide average implant duration is 80 to 100 days, and maximum duration on support has exceeded 2 years. The probability of device failure has been shown to be $35 \%$ at 2 years.

The HeartMate XVE (Thoratec Corp. Pleasanton, Calif), a pulsatile pump, can be operated in either a fixed-rate (partial support) or automatic mode (full support), and it can produce a maximum stroke volume of $83 \mathrm{ml}$ at varying rates (from 50 to 120 beats/min), resulting in flow rates from 4 to $10 \mathrm{l} / \mathrm{min}$ (Figure 1). The pulsatile flow is created using a pusher plate system [25]. In automatic mode, the pump senses when the chamber is full and activates the pusher plate. In case of an emergency, a portable hand pump can be used to activate the device. The patient's body size is an important factor in allowing device placement. The size of the device requires patients to have a body surface area of more than $1.5 \mathrm{~m}^{2}$. The device is made of a titanium alloy external housing with inflow and outflow tracts that utilize porcine xenograft valves $(25 \mathrm{~mm})$. The unique characteristic of the device is its internal bloodcontacting surface, which is made on one side of textured titanium and on the other of textured polyurethane. This textured surface encourages the deposition of a fibrin-cellular matrix that forms a pseudoneointima. The formation of this surface greatly reduces the need for anticoagulation because thrombus formation is greatly reduced [26]. Patients with these devices take aspirin (primarily as an anti-inflammatory, not as an anticoagulant) as their only anticoagulation with a subsequent low rate of thromboembolic complications (7\%) [11, 25-27]. The HeartMate VE was used in the Randomized Evaluation of Mechanical Assistance for the Treatment of Congestive Heart Failure (REMATCH) trial to compare medical and circulatory assist device treatments for end-stage heart failure. Patients with this device showed better results than the medically treated group. However, the survival in the VAD group after 2 years was $28 \%$, compared to only $8 \%$ in the medically treated patients [11]. This trial established the substantial risk of mechanical failure and device-related complications inherent in the first-generation pulsatile devices $[11,28]$.

2.2. Second Generation of LVAD Types. The engineering of continuous-flow rotary pump technology represents a milestone and novel design concept for LVADs. These devices have now largely replaced the use of the firstgeneration of pulsatile, volume displacement pumps. The second-generation rotary pumps have the advantage of a smaller design and potential for greater long-term mechanical reliability by eliminating the reservoir chamber and valves needed for first-generation pulsatile pumps [10]. The second-generation rotary blood pumps are typically with an "axial" blood flow path, which have an internal rotor within the blood flow path that is suspended by contact bearings. In comparison, third-generation pumps have generally been used to categorize continuous-flow rotary devices with an impeller or rotor suspended in the blood flow path using a "noncontact" bearing design.

The most common implantable pumps are the HeartMate II (Thoratec Corp, Pleasanton, CA, USA), and the Jarvik 2000 (Jarvik Heart Inc.) [16, 17, 22, 29]. Up to date, the HeartMate II is the most successful second-generation pump worldwide and approved as bridge to transplant and as destination therapy [4-7].

The HeartMate II LVAD is an axial flow pump that had its origin in the early 1990s (Figure 2) [30]. This is an axial-flow rotary LVAD made of titanium with a pump implant volume of $63 \mathrm{ml}$. The HeartMate II contains a rotor capable of producing flow rates greater than $10 \mathrm{~L} / \mathrm{min}$ at resolutions (RPM) ranging from 8,000 to 15,000 (Figure 2) [10]. The inflow cannula is connected to the apex of the left ventricle, with the outflow graft connected to the ascending aorta. Like the other axial-flow pumps, there is a risk of generating negative intraventricular pressure and collapsing the ventricle. As a result, inflow cannula positioning and ventricular preload are important. The intraventricular portion of the inflow tract has been elongated and as a result, tends to stent open the middle of the ventricle, thus improving reliability of continuous flow throughout the cardiac cycle [30]. Anticoagulation is at present recommended to keep INR between 1.5 and 2.5 [31]. The pump is inserted preperitoneally or within the abdominal musculature. Power and control are supplied through a percutaneous lead that is attached to a controller that can be connected to rechargeable batteries worn by the patient or to an external power module. The system is operated at a fixed rotational speed set by the clinician [30].

2.3. Third Generation of LVAD Types. Levitation systems utilized in third-generation rotary blood pumps suspend the moving impeller within the blood field without any mechanical contact. The magnetic and/or hydrodynamic levitation of the impeller without any contact bearings with the pump is the major advancement of the thirdgeneration pumps. These designs have the potential to significantly improve device durability, but it should be noted 


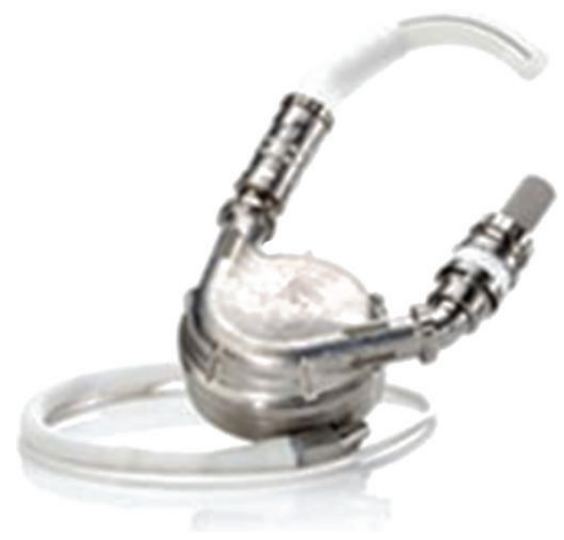

(a)

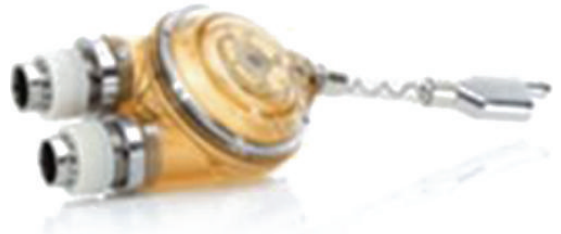

(b)

FIgURE 1: First generation pulsatile left ventricular assist device HeartMate XVE (a) and Thoratec paracorporeal device (b).

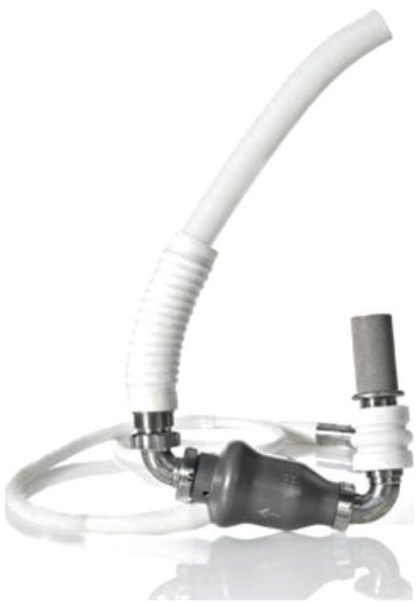

(a)

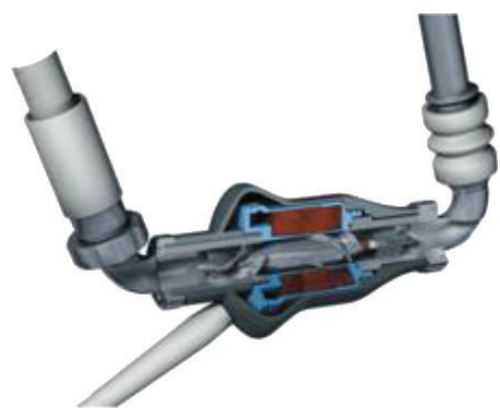

(b)

FIgURE 2: HeartMate II left ventricular assist device and cross-sectional internal view (Illustration Thoratec Corporation). Reprinted with permission from Thoratec Corporation.

that blood immersed bearings such as in the HeartMate II have been reported to have almost imperceptible wear, and with estimated life well in excess of 15 years [32]. Pump design can be further distinguished by utilization of hydrodynamic levitation only (VentrAssist; Ventracor Ltd., Sydney, Australia), and hydrodynamic levitation working in synergy with magnetic levitation for suspension (HVAD Pump, HeartWare, Inc.; Levacor; World Heart Corp.), or full magnetic suspension such as the HeartMate III, Thoratec Corp. [10, 18, 33-35]. The Berlin Heart Incor (Berlin Heart AG) [36] and the DuraHeart (Terumo Somerset, USA) are both magnetic levitation pumps and also assigned to 3rd generation pumps $[9,18]$.

The HVAD Pump, part of the HeartWare Ventricular Assist System (HeartWare Inc), is a small centrifugal flow pump with a displacement volume of $50 \mathrm{ml}$ and an output capacity of $10 \mathrm{~L} / \mathrm{min}$ (Figure 3 ). A unique wideblade impeller is suspended by hybrid passive magnets and hydrodynamic forces. There are no points of mechanical contact within the pump, effectively ensuring a "wearless" system. The design integrates two motor stators for singlemotor fault protection to increase reliability. An integrated inflow cannula is inserted into the left ventricle and is held in position by an adjustable sewing ring; the pump is positioned in the pericardial space. The $10-\mathrm{mm}$ outflow graft is anastomosed to the ascending aorta. External system components include the microprocessor-based controller, a monitor, lithium-ion battery packs, alternating current and direct current power adapters, and a battery charger. Physiologic control algorithms are incorporated for safe operation. Preclinical life cycle tests have shown the HVAD to be highly reliable. This system design offers reliability, portability, and ease of use for ambulatory patients [37]. The device size and the integrated inflow cannula allow it to be implanted completely in the pericardial space, directly adjacent to the heart, thereby avoiding the abdominal surgery generally required to implant competing devices. Reduced procedural invasiveness is expected to lead to more 


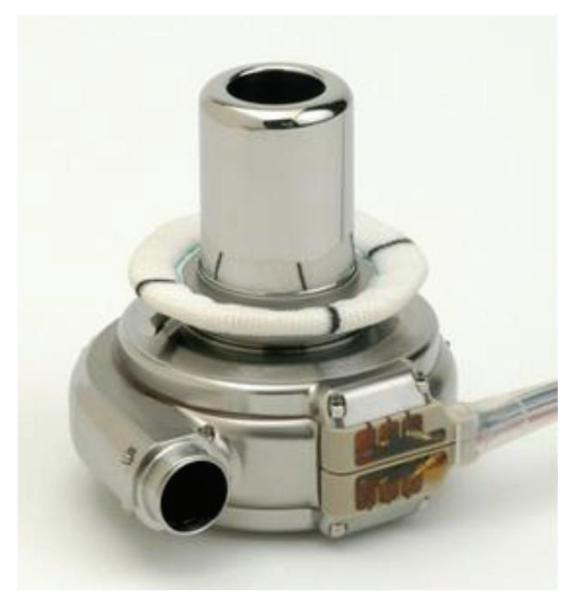

(a)

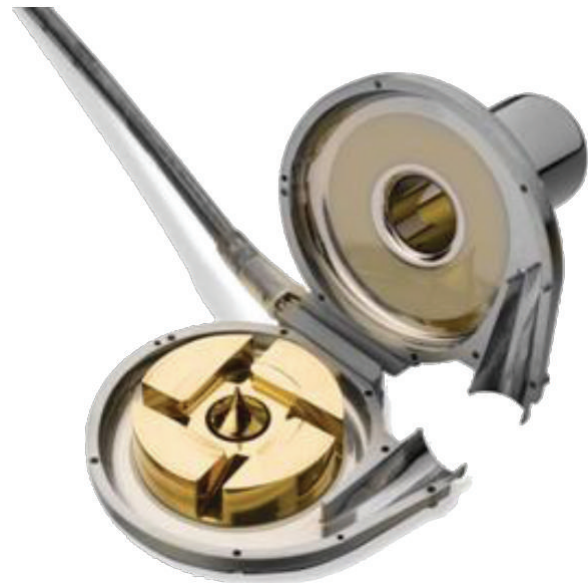

(b)

Figure 3: External (a) and internal (b) view of third-generation continuous-flow rotary left ventricular assist device HVAD (HeartWare). Reprinted with permission from HeartWare.

rapid postoperative recovery and improved patient outcomes [12]. Additionally, the biventricular use has been described successfully, but is as yet an "off-label" use [13]. In such a case, additional modifications like pulmonary artery banding or crimping of the outflow cannula may be necessary to increase the afterload for the right ventricular assist $[13,38]$.

2.4. Contemporary Outcomes. A patient with end-stage heart failure (who is not a transplant candidate) today faces two contrasting options. The first rests with the traditional approach of optimal medical therapy which would involve: (1) severe debility resulting in overwhelming fatigue and precluding any form of physical activity, (2) frequent hospitalizations to treat acute decompensations, and (3) a twoyear survival rate under $15 \%[1-3,11]$. The second option involves implantation of an LVAD with a two-year survival rate last reported to be $70 \%$ for implants in 2007 and early 2008 (but likely well above 70\% for implants performed today), as well as substantial improvements in quality of life as these patients are typically able to return to normal daily activities [4-10, 12, 14-17].

In support, see Figure 4 for a summary of survival data for patients enrolled in pivotal US DT trials treated with Thoratec's current-generation HeartMate II, priorgeneration HeartMate XVE, and optimal medical therapy $[4,5,7,11]$.

As shown in Figure 4, 2-year survival with optimal medical therapy was approximately $8 \%$ for patients between 1998 and 2001. Unfortunately, there have been no major improvements in the care of heart failure patients since then, suggesting that survival with medical therapy has not changed appreciably. This factor is further supported by the observation that there was no difference in survival for patients implanted with the HeartMate XVE from 1998-2001 when compared with those implanted 2005-2007 [6].

Pulsatile, volume displacement devices have several limitations inherent in their engineering design that preclude

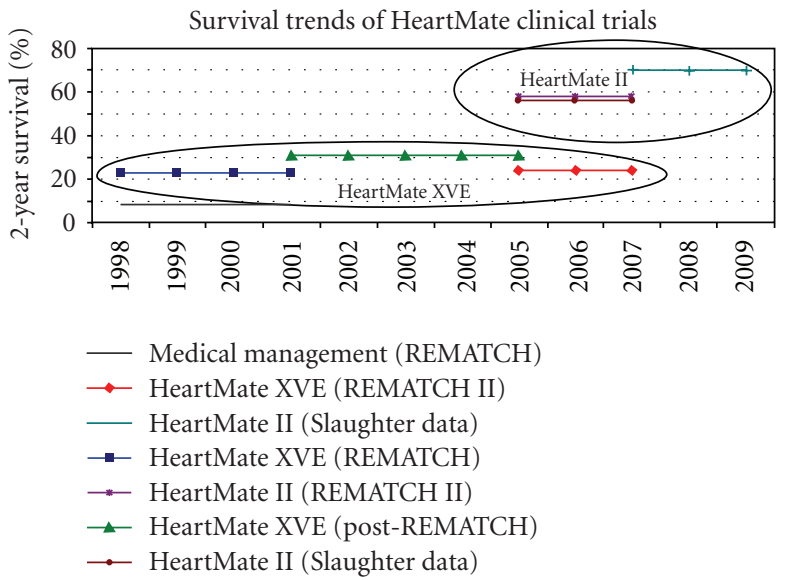

Figure 4: Two-year survival trends of HeartMate clinical trials. Sources: ISHLT, Slaughter [4], Rogers [5], Pagani [7], and Rose [11].

their practical use for long-term circulatory support. The most critical constraint of the majority of these devices has been the incidence of pump malfunctions resulting in death or need for reoperations [15]. The landmark REMATCH trial showed for the HeartMate XVE a 35\% failure rate after 2 years, with a mortality of more than $10 \%$ [11]. The rate of serious infection, including the pump itself, the pump pocket, and the driveline range between 18-80\% after LVAD placement.

For the first-generation VADs, the published range of thromboembolic events reaches from 5 to $50 \%$ depending on the different LVAD types and variation in anticoagulation regimes. The lowest thromboembolic rates have been published for the HeartMate XVE (3-9\%) [11].

Reports from the second-generation rotary pumps have demonstrated efficacy in providing hemodynamic support, favourable risk to benefit assessment, and improved mechanical performance $[4,7,10,16]$. Up to date, the HeartMate 

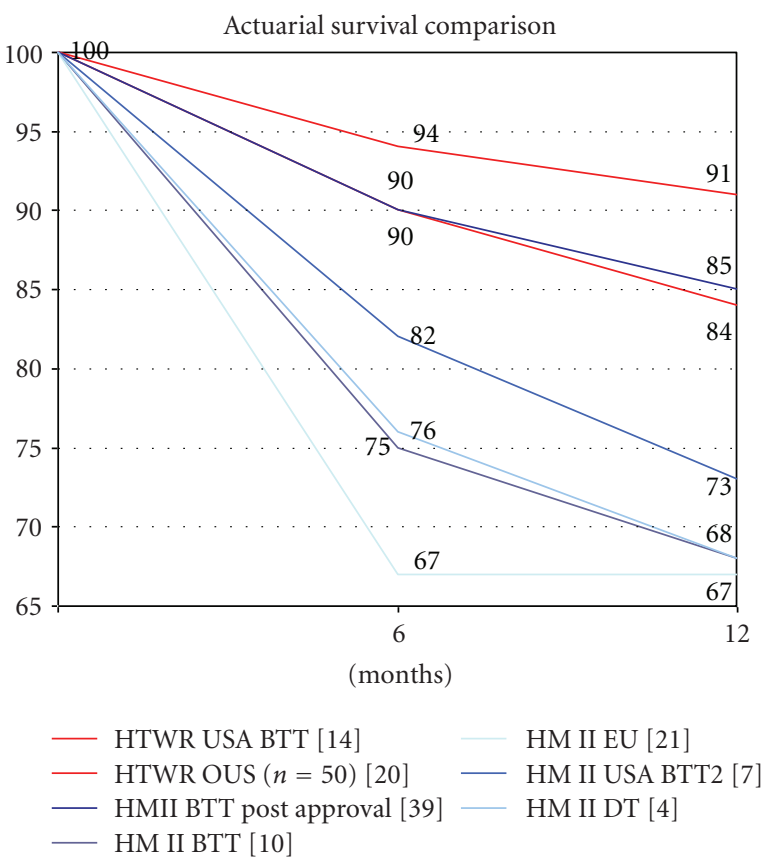

Figure 5: Actuarial survival comparison between HeartMate studies and HVAD assist device. (1) Results from the US BTT Trial presented at AHA Meeting in November by Aaronson [14], (2) Strüber et al. 2010 [20], (3) Miller et al. 2007 [10], (4) HeartMate II Post-approvel study [39], (5) Strüber et al. 2008 [21], (6) Pagani et al. 2009 [7], (7) Slaughter et al. 2009 [4].

II is the most successful second-generation LVAD with over 6000 implants worldwide and 1500 implants in Europe. The reported survival rates have improved as experienced was gained from the initial clinical trial results [10] to the postapproval study [39] from $89 \%$ to $96 \%$ (30 days), from $75 \%$ to $90 \%$ (6 months), and from $68 \%$ to $85 \%$ (1 year) (Figures 4 and 5).

Additionally, results from clinical studies also have shown early improvements followed by long-term stability of renal and hepatic function, as well as limited adverse effects on neurocognitive performance [6, 10, 17]. These improvements have led to increased acceptance of LVAD therapy for long-term support.

At the ISHLT conference in April 2010, Mark Slaughter from the University of Louisville presented data on 93 patients implanted with the HeartMate II for Destination Therapy at two centers (University of Louisville and Duke) from 2005 to 2009 . This data showed that patients implanted during 2007-2009 had a 2-year survival rate of 70\%, a substantial improvement over the $58 \%$ survival rate observed in the HeartMate II's pivotal trial in destination therapy (DT), which enrolled patients from 2005-2007. The important conclusion of this study is survival rates are continuing to improve as surgeons gain experience in patient selection, operative techniques, and postop care.

The incidence of thromboembolic events is relatively low for the HeartMate II and ranges from 3 to 6 events per 100 patient-years [4]. In the randomized destination therapy trial of the HeartMate II versus the HeartMate XVE, the hemorrhagic and ischemic stroke rates (at 0.06 and 0.07 events per patient-year) tended to be lower in comparison to HeartMate XVE (at 0.10 and 0.12 events per patient-year) [4]. Other second-generation devices (DuraHeart, VentrAssist) show somewhat higher incidence of neurological complications $[9,40]$. The incidence of driveline and pump infection is still remarkable, ranging from 13 to $27 \%[6,10,17,21,28]$. However the lowest rate is reported for the HeartMate II [19]. In comparison to severe device-related infections with firstgeneration pumps, those with second-generation devices seldom lead to fatal outcomes $[6,28]$.

Of note, while the survival data outlined above only involves patients implanted with second-generation pumps, we currently do not expect any major differences in the 2year survival among any of the later stage devices. In support, we note that data from HeartWare's European BTT trial (third-generation) appears as good as the data from Thoratec's US BTT trial (Figure 5) [12]. Additionally, at the 2010 AHA scientific meeting, initial results from the company's U.S. Bridge-to-Transplant clinical trial (ADVANCE) were presented. This was designed as a noninferiority trial and the data showed survival to transplant or ongoing support of $92 \%$ after 180 days (Table 1) [14]. The observed survival rates were similar to $90 \%$ for a control group of commerciallyavailable devices including not only HeartMate II, but also patients with pulsatile flow XVE and Thoratec IVADs. The functional capacity and quality of life were improved at three month after placement, as with approved pumps [14]. In Figure 5 the actuarial survival of the most currently used second (HeartMate II) and third-generation (HVAD) rotary blood pumps are highlighted. However, whether the thirdgeneration of rotary blood pumps will result in significant improvements in clinical outcomes over those observed with the second-generation of rotary pumps with axial design is not known at this time.

The currently reported HVAD-related infection rate ranges from $10.7 \%$ (driveline exit) to $6.4 \%$ (sepsis). In the study cohort, no pump pocket infection was seen [14]. The observed stroke rates were 0.11 events per patient-year for ischemic and 0.05 for hemorrhagic stroke, respectively [14], compared to 0.09 and 0.05 in the HeartMate II clinical trial [7], and 0.05 and 0.01 in the more recent HeartMate II post approval study [4]. 50\% of ischemic strokes in the HVAD trial occurred within 48 hours following device implantation [14].

Quality-of-life improvements with LVAD therapy can be dramatic. In support, we note an early study of the HeartMate II in the Bridge-to-Transplant context which showed that $43 \%$ of patients improved from NYHA Class IV (symptomatic at rest: mostly bed-bound) to Class I (no symptoms and no limitation in ordinary physical activity), while another $43 \%$ improved from Class IV to Class II (mild symptoms and slight limitation on activity) or Class III (marked limitations; comfortable only at rest) [10].

While the number of quality of life focused studies remains somewhat limited, recent data includes presentations from ACC and ISHLT 2010. For example, one study examined three quality of life measurements from the 
TABLE 1: Survival after HVAD Implantation: Evaluation of HeartWare HVAD left ventricular assist device for the treatment of advanced heart failure: Results of the ADVANCE Bridge to transplant Trial.

\begin{tabular}{lc}
\hline \multicolumn{2}{c}{ ADVANCE Trial Outcomes at 180 days* } \\
\hline Transplant or alive & $92.0 \%$ \\
Alive & $62.8 \%$ \\
Transplant & $29.2 \%$ \\
Device exchange & $4.4 \%$ \\
Death & $3.6 \%$ \\
\hline
\end{tabular}

* Presentation at the AHA meeting 2010 [14].

HeartMate II BTT and DT trial, including the Minnesota Living with Heart Failure (MLWHF), Kansas City Cardiomyopathy Questionnaires (KCCQ) and 6-minute walk distance (6MWD). The study found significant improvements in all QoL measurements from baseline to 6 months. The presentation also highlighted that even greater improvements are possible in the Destination Therapy population compared to BTT patients (39\% improvement in DT versus BTT patients, $P=.003)$, likely due to their generally sicker status [4-6].

Indeed, from the published clinical experience, patients themselves will find quality of life benefits to be a more attractive justification for LVAD implantation than even improvements in survival. As a result, these observed benefits may provide a significant driver for device uptake $[4,5]$.

\section{Patient Selection}

One of the most important aspects of device implantation is patient selection. Heart failure in some form must be present. Signs of failure such as pulmonary capillary wedge pressure higher than $20 \mathrm{~mm} \mathrm{Hg}$, cardiac index less than $2.0 \mathrm{~L} / \mathrm{min} / \mathrm{m}^{2}$, or systolic blood pressure below $80 \mathrm{~mm} \mathrm{Hg}$ despite best medical management should be present [10]. Columbia University and the Cleveland Clinic Foundation devised a scoring system in 1995 to predict which patients would have a successful outcome after LVAD implantation [41]. However, as the technology evolved, it widened and extended the use of these devices and the Columbia score was revised to better reflect the current LVAD-eligible population [42]. The previous score utilized 10 factors found to be significant for mortality using univariate analysis with a score higher than 5 corresponding to more than a 33\% risk of postimplantation death [41]. The revised score was based on 130 patients receiving vented electric HeartMate devices from 1996 to 2001. Univariate and multivariate analyses were performed to determine operative mortality. The new preoperative risk factors predicting mortality by univariate analysis are: previous LVAD/RVAD (right ventricular assist device), acute myocardial infarction, postcardiotomy, central venous pressure (CVP) greater than $16 \mathrm{~mm} \mathrm{Hg}$, prothrombin time (PT) greater than 16 seconds, preoperative ventilation, redo-surgery, coronary artery disease, and dilated cardiomyopathy. Interestingly, preoperative renal insufficiency was not found to impact survival in the new scoring system,
TABLE 2: Clinically used adult LVAD types.

\begin{tabular}{|c|c|c|c|}
\hline & $\begin{array}{l}\text { First-generation } \\
\text { LVADs }\end{array}$ & $\begin{array}{l}\text { Second-generation } \\
\text { LVADs }\end{array}$ & $\begin{array}{l}\text { Third-generation } \\
\text { LVADs }\end{array}$ \\
\hline $\begin{array}{l}\text { Pump } \\
\text { design }\end{array}$ & Pulsatile flow & $\begin{array}{l}\text { Continuous-flow } \\
\text { (axial pump) }\end{array}$ & $\begin{array}{l}\text { Continuous-flow } \\
\text { (centrifugal pump) }\end{array}$ \\
\hline $\begin{array}{l}\text { LVAD } \\
\text { types }\end{array}$ & $\begin{array}{l}\text { HeartMate XVE } \\
\text { Novacor LVAS } \\
\text { Thoratec LVAD }\end{array}$ & $\begin{array}{l}\text { HeartMate II } \\
\text { Jarvik FlowMaker } \\
\text { Incor Berlin Heart }\end{array}$ & $\begin{array}{l}\text { HeartWare LVAD } \\
\text { DuraHeart LVAS }\end{array}$ \\
\hline
\end{tabular}

unlike the old system. This is likely due to aggressive treatment of renal insufficiency with ultrafiltration and hemodialysis. A stepwise linear regression model identified a ventilated patient and a previous LVAD as independent predictors of mortality following device insertion [42, 43]. After multivariate analysis the 5 new factors included in the scoring system were: ventilated patients (score of 4 ), redo surgery (score of 2), previous LVAD inserted (score of 2), CVP higher than $16 \mathrm{~mm} \mathrm{Hg}$ (score of 1), and PT higher than 16 seconds (score of 1). A score higher than 5 corresponds with a $47 \%$ mortality, compared with $9 \%$ mortality for a score lower than 5 . The positive and negative predictive value of this scoring system is $79 \%$ and $70 \%$, respectively [42].

The Seattle Heart Failure Model (SHFM) is a prospective validated multivariate risk model and a tool that predicts survival of heart failure patient $[44,45]$. This score may facilitate identifications of high-risk patients to evaluate for potential LVAD therapy, by providing an estimate of 1- to 5-year outcome with medical therapy [43]. The SHFM, with the addition of inotropic use, mechanical support (intraaortic ballon pump), and ventilation, is able to successfully provide risk stratification in patients considered for potential LVAD therapy [43].

The study by Holman et al. identified preoperative risk factors for mortality based on the results of INTERMACS registry (registry of FDA approved durable mechanical circulatory assist device). In this analysis older age (relative risk $=1.41 ; P<.001$ ), ascites (relative risk $=2.04 ; P=.003$ ), increased bilirubin (relative risk $=1.49 ; P<.05$ ), and cardiogenic shock (INTERMACS level 1) (relative risk $=1.59 ; P=$ .02 ) are highly associated with postimplant mortality [28].

The urgency of device placement has also been shown to play a factor in survival. In a study by Deng et al. patients receiving emergent LVADs had a lower survival to transplantation rate than those receiving devices urgently or those who did not need devices [46]. However, electively implanted LVAD patients with no subsequent transplantation had better survival than medically treated heart failure patients who also did not get transplanted. This occurred despite the fact that the LVAD recipients were a sicker group of patients [46].

\section{Summary}

The shortage of appropriate donor organs and the expanding pool of patients waiting for heart transplantation have led to growing interest in alternative strategies, particularly in 
TABLE 3: Most commonly used LVADs.

\begin{tabular}{|c|c|c|}
\hline LVAD Types & HeartWare System & HeartMate II \\
\hline $\begin{array}{l}\text { Support duration } \\
\text { (patient years) }\end{array}$ & $47.8[20]$ & $211[5]$ \\
\hline \multicolumn{3}{|l|}{ Outcome } \\
\hline (i) 30 days survival & $98 \%[14]$ & $96 \%[39]$ \\
\hline (ii) 1-year survival & $91 \%[14]$ & $85 \%[39]$ \\
\hline (iii) 2-year survival & - & $70 \%[4]$ \\
\hline Quality of life & $+++[14]$ & $+++[5,10]$ \\
\hline \multicolumn{3}{|l|}{ Approval } \\
\hline (i) BTT & Yes & Yes \\
\hline (ii) DT & No, but on the way & Yes \\
\hline \multirow{5}{*}{ Advantages } & $\begin{array}{l}\text { (i) Miniaturized } \\
\text { pump }\end{array}$ & $\begin{array}{l}\text { (i) Long-term } \\
\text { support }\end{array}$ \\
\hline & $\begin{array}{l}\text { (ii) Implanted in } \\
\text { the intrapericardial } \\
\text { space }\end{array}$ & $\begin{array}{l}\text { (ii) } \\
\text { Destination } \\
\text { therapy }\end{array}$ \\
\hline & $\begin{array}{l}\text { (iii) Indicated to } \\
\text { support patients } \\
\text { with } B S A \geq 1.2 \mathrm{~m}^{2}\end{array}$ & $\begin{array}{l}\text { (iii) } \\
\text { Durability }\end{array}$ \\
\hline & $\begin{array}{l}\text { (iv) Right } \\
\text { ventricular } \\
\text { implantation }\end{array}$ & $\begin{array}{l}\text { (iv) More } \\
\text { than } 1500 \\
\text { implants in } \\
\text { Europe }\end{array}$ \\
\hline & $\begin{array}{l}\text { (v) Biventricular } \\
\text { implantation }\end{array}$ & \\
\hline \multirow{7}{*}{ Disadvantages } & (i) Driveline & $\begin{array}{l}\text { (i) Require a } \\
\text { pump pocket }\end{array}$ \\
\hline & (a) Infection & (ii) Driveline \\
\hline & (b) Breaking & $\begin{array}{c}\text { (a) } \\
\text { Infection }\end{array}$ \\
\hline & (ii) Neurological & \\
\hline & dysfunction & Breaking \\
\hline & (iii) Hemolysis & $\begin{array}{l}\text { (iii) } \\
\text { Neurological } \\
\text { dysfunction }\end{array}$ \\
\hline & & (iv) Bleedings \\
\hline
\end{tabular}

BTT: bridge to transplant; DT: destination therapy; BSA: body surface area.

mechanical circulatory support. The spectrum of VAD therapy is clearly expanding. The second and third-generation of nonpulsatile continuous-flow ventricular assist devices have yielded encouraging preliminary data suggesting improved outcomes, quality of life, and device durability. Table 2 shows the typical classification of adult LVADs and Table 3 highlights the outcome, complications and characteristics of the most commonly used LVADs. The development of validated risk stratification models will lead to improved patient selection and timing of device implant, with overall improved outcomes over time. However, clinical trials are needed to demonstrate the potential and superiority of these promising therapies. Further minimally invasive surgical implantation techniques in combination with newer miniaturized pumps may reduce the operative risk, especially in the elderly. The development of fully implantable LVAD technology that incorporates an implantable battery with transcutaneous energy transfer (TET) may also lead to reduced infection rate (infection reduction technology).

\section{Acknowledgment}

The authors have no financial associations or relationship with industry that might pose a conflict of interest with the submitted manuscript.

\section{References}

[1] American Heart Association. 2009. Heart and Stroke facts: 2006 update, http://www.americanheart.org.

[2] Br. Heart Found. 2009, http://www.heartstats.org.

[3] E. Braunwald, D. P. Zipes, P. Libby, and R. O. Bonow, Braunwald's Heart Disease: A Textbook of Cardiovascular Medicine, Saunders, Philadelphia, Pa, USA, 7th edition, 2005.

[4] M. S. Slaughter, J. G. Rogers, C. A. Milano et al., "Advanced heart failure treated with continuous-flow left ventricular assist device," The New England Journal of Medicine, vol. 361, no. 23, pp. 2241-2251, 2009.

[5] J. G. Rogers, K. D. Aaronson, A. J. Boyle et al., "Continuous flow left ventricular assist device improves functional capacity and quality of life of advanced heart failure patients," Journal of the American College of Cardiology, vol. 55, no. 17, pp. 1826$1834,2010$.

[6] J. K. Kirklin, D. C. Naftel, R. L. Kormos et al., "Second INTERMACS annual report: more than 1,000 primary left ventricular assist device implants," Journal of Heart and Lung Transplantation, vol. 29, no. 1, pp. 1-10, 2010.

[7] F. D. Pagani, L. W. Miller, S. D. Russell et al., "Extended mechanical circulatory support with a continuous-flow rotary left ventricular assist device," Journal of the American College of Cardiology, vol. 54, no. 4, pp. 312-321, 2009.

[8] F. D. Pagani, "Continuous-flow rotary left ventricular assist devices with "3rd Generation" design," Seminars in Thoracic and Cardiovascular Surgery, vol. 20, no. 3, pp. 255-263, 2008.

[9] M. Morshuis, A. El-Banayosy, L. Arusoglu et al., "European experience of DuraHeart ${ }^{\mathrm{TM}}$ magnetically levitated centrifugal left ventricular assist system," European Journal of CardioThoracic Surgery, vol. 35, no. 6, pp. 1020-1028, 2009.

[10] L. W. Miller, F. D. Pagani, S. D. Russell et al., "Use of a continuous-flow device in patients awaiting heart transplantation," The New England Journal of Medicine, vol. 357, no. 9, pp. 885-896, 2007.

[11] E. A. Rose, A. C. Gelijns, A. J. Moskowitz et al., "Long-term use of a left ventricular assist device for end-stage heart failure," The New England Journal of Medicine, vol. 345, no. 20, pp. 1435-1443, 2001.

[12] G. M. Wieselthaler, G. O'Driscoll, P. Jansz, A. Khaghani, and M. Strueber, "Initial clinical experience with a novel left ventricular assist device with a magnetically levitated rotor in a multi-institutional trial," Journal of Heart and Lung Transplantation, vol. 29, no. 11, pp. 1218-1225, 2010.

[13] R. Hetzer, T. Krabatsch, A. Stepanenko, E. Hennig, and E. V. Potapov, "Long-term biventricular support with the heartware implantable continuous flow pump," Journal of Heart and Lung Transplantation, vol. 29, no. 7, pp. 822-824, 2010.

[14] K Aaronson, "Evaluation of HeartWare HVAD left ventricular assist device system for the treatment of advanced heart failure: results of the ADVANCE Bridge to transplant Trial," Presentation at the AHA meeting, November 2010. 
[15] R. D. Dowling, S. J. Park, F. D. Pagani et al., "HeartMate VE LVAS design enhancements and its impact on device reliability," European Journal of Cardio-Thoracic Surgery, vol. 25, no. 6, pp. 958-963, 2004.

[16] M. P. Siegenthaler, O. H. Frazier, F. Beyersdorf et al., "Mechanical reliability of the Jarvik 2000 Heart," Annals of Thoracic Surgery, vol. 81, no. 5, pp. 1752-1759, 2006.

[17] O. H. Frazier, T. J. Myers, S. Westaby et al., "Use of the Jarvik 2000 left ventricular assist system as a bridge to heart transplantation or as destination therapy for patients with chronic heart failure," Annals of Surgery, vol. 237, no. 5, pp. 631-637, 2003.

[18] C. Nojiri, T. Kijima, J. Maekawa et al., "Development status of terumo implantable left ventricular assist system," Artificial Organs, vol. 25, no. 5, pp. 411-413, 2001.

[19] J. Lahpor, A. Khaghani, R. Hetzer et al., "European results with a continuous-flow ventricular assist device for advanced heartfailure patients," European Journal of Cardio-Thoracic Surgery, vol. 37, no. 2, pp. 357-361, 2010.

[20] M. Strüber, G. O’Drscoli, P. Jansz et al., "Multicenter evaluation of an intrapericardial left ventricular assist system," Journal of the American College of Cardiology, vol. 57, no. 12, pp. 1375-1382, 2011.

[21] M. Strüber, K. Sander, J. Lahpor et al., "HeartMate II left ventricular assist device; early European experience," European Journal of Cardio-Thoracic Surgery, vol. 34, no. 2, pp. 289-294, 2008.

[22] S. Haj-Yahia, E. J. Birks, P. Rogers et al., "Midterm experience with the Jarvik 2000 axial flow left ventricular assist device," Journal of Thoracic and Cardiovascular Surgery, vol. 134, no. 1, pp. 199-203, 2007.

[23] O. H. Frazier, E. A. Rose, P. McCarthy et al., "Improved mortality and rehabilitation of transplant candidates treated with a long-term implantable left ventricular assist system," Annals of Surgery, vol. 222, no. 3, pp. 327-338, 1995.

[24] O. H. Frazier, E. A. Rose, M. C. Dz et al., "Multicenter clinical evaluation of the HeartMate vented electric left ventricular assist system in patients awaiting heart transplantation," Journal of Thoracic and Cardiovascular Surgery, vol. 122, no. 6, pp. 1186-1195, 2001.

[25] V. L. Poirier, "Worldwide experience with the TCI HeartMate system: issues and future perspective," The Thoracic and Cardiovascular Surgeon, vol. 47, pp. 316-320, 1999.

[26] B. C. Sun, K. A. Catanese, T. B. Spanier et al., "100 Longterm implantable left ventricular assist devices: the columbia presbyterian interim experience," Annals of Thoracic Surgery, vol. 68, no. 2, pp. 688-694, 1999.

[27] P. M. McCarthy, N. O. Smedira, R. L. Vargo et al., "One hundred patients with the heartmate left ventricular assist device: evolving concepts and technology," Journal of Thoracic and Cardiovascular Surgery, vol. 115, no. 4, pp. 904-912, 1998.

[28] W. L. Holman, R. L. Kormos, D. C. Naftel et al., "Predictors of death and transplant in patients With a mechanical circulatory support device: a multi-institutional study," Journal of Heart and Lung Transplantation, vol. 28, no. 1, pp. 44-50, 2009.

[29] K. Nawata, T. Nishimura, and S. Kyo, "Outcomes of midterm circulatory support by left ventricular assist device implantation with descending aortic anastomosis," Journal of Artificial Organs, vol. 13, no. 4, pp. 197-201, 2011.

[30] B. P. Griffith, R. L. Kormos, H. S. Borovetz et al., "HeartMate II left ventricular assist system: from concept to first clinical use," Annals of Thoracic Surgery, vol. 71, no. 3, supplement 1, pp. S116-S120, 2001.
[31] A. J. Boyle, S. D. Russell, J. J. Teuteberg et al., "Low thromboembolism and pump thrombosis with the heartMate II left ventricular assist device: analysis of outpatient anticoagulation," Journal of Heart and Lung Transplantation, vol. 28, no. 9, pp. 881-887, 2009.

[32] S. H. Reichenbach, K. B. Masterson, K. C. Butler, and D. J. Farrar, "Negligible bearing wear in explanted heartMate II LVADs following clinical support for up to four years," in Proceedings of the Annual Meeting of the International Society of Rotary Blood Pumps, Berlin, Germany, November 2010.

[33] H. Hoshi, T. Shinshi, and S. Takatani, "Third-generation blood pumps with mechanical noncontact magnetic bearings," Artificial Organs, vol. 30, no. 5, pp. 324-338, 2006.

[34] D. J. Farrar, K. Bourque, C. P. Dague, C. J. Cotter, and V. L. Poirier, "Design features, developmental status, and experimental results with the heartmate III centrifugal left ventricular assist system with a magnetically levitated rotor," ASAIO Journal, vol. 53, no. 3, pp. 310-315, 2007.

[35] G. B. Bearnson, G. B. Jacobs, J. Kirk, P. S. Khanwilkar, K. E. Nelson, and J. W. Long, "HeartQuest ventricular assist device magnetically levitated centrifugal blood pump," Artificial Organs, vol. 30, no. 5, pp. 339-346, 2006.

[36] C. Schmid, M. Jurmann, D. Birnbaum et al., "Influence of inflow cannula length in axial-flow pumps on neurologic adverse event rate: results from a multi-center analysis," Journal of Heart and Lung Transplantation, vol. 27, no. 3, pp. 253-260, 2008.

[37] J. A. LaRose, D. Tamez, M. Ashenuga, and C. Reyes, "Design concepts and principle of operation of the heartware ventricular assist system," ASAIO Journal, vol. 56, no. 4, pp. 285-289, 2010.

[38] M. Strueber, A. L. Meyer, D. Malehsa, and A. Haverich, "Successful use of the HeartWare HVAD rotary blood pump for biventricular support," Journal of Thoracic and Cardiovascular Surgery, 2010.

[39] R. C. Starling, Y Naka, and A. J. Boyle, "Results of the postFDA-approval study with a continuous flow left ventricular assist device as a bridge to heart transplantation: a prospective study using the INTERMACS registry. Presented at heart failure society of America," Journal of the American College of Cardiology. In press.

[40] D. Esmore, D. Kaye, P. Spratt et al., "A prospective, multicenter trial of the ventrAssist left ventricular assist device for bridge to transplant: safety and efficacy," Journal of Heart and Lung Transplantation, vol. 27, no. 6, pp. 579-588, 2008.

[41] M. C. Oz, D. J. Goldstein, P. Pepino et al., "Screening scale predicts patients successfully receiving long-term implantable left ventricular assist devices," Circulation, vol. 92, no. 9, pp. II169-II173, 1995.

[42] V. Rao, M. C. Oz, M. A. Flannery, K. A. Catanese, M. Argenziano, and Y. Naka, "Revised screening scale to predict survival after insertion of a left ventricular assist device," Journal of Thoracic and Cardiovascular Surgery, vol. 125, no. 4, pp. 855-862, 2003.

[43] W. C. Levy, D. Mozaffarian, D. T. Linker, D. J. Farrar, and L. W. Miller, "Can the seattle heart failure model be used to riskstratify heart failure patients for potential left ventricular assist device therapy?" Journal of Heart and Lung Transplantation, vol. 28, no. 3, pp. 231-236, 2009.

[44] D. Mozaffarian, S. D. Anker, I. Anand et al., "Prediction of mode of death in heart failure: The Seattle Heart Failure Model," Circulation, vol. 116, no. 4, pp. 392-398, 2007. 
[45] L. W. Miller, "Patient selection for the use of ventricular assist devices as a bridge to transplantation," Annals of Thoracic Surgery, vol. 75, no. 6, supplement 1, pp. S66-S71, 2003.

[46] M. C. Deng, M. Weyand, D. Hammel et al., "Selection and management of ventricular assist device patients: the muenster experience," Journal of Heart and Lung Transplantation, vol. 19, no. 8, pp. S77-S82, 2000. 


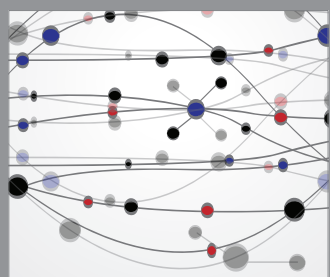

The Scientific World Journal
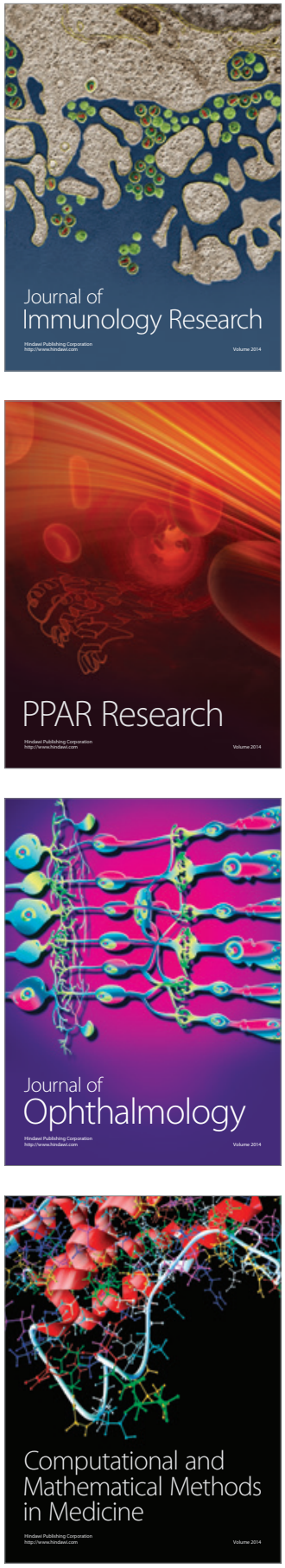

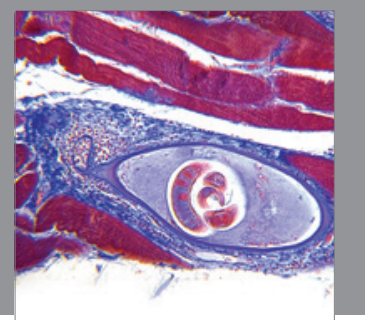

Gastroenterology

Research and Practice
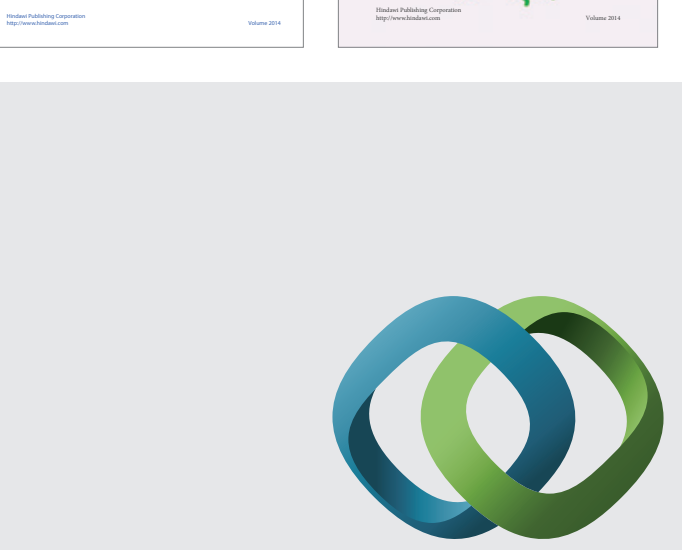

\section{Hindawi}

Submit your manuscripts at

http://www.hindawi.com
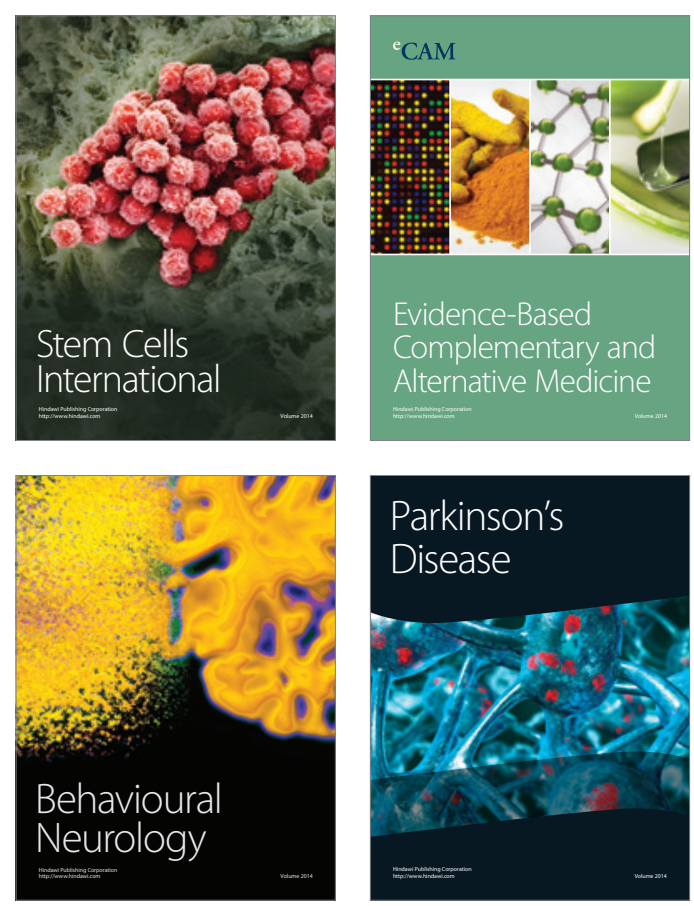

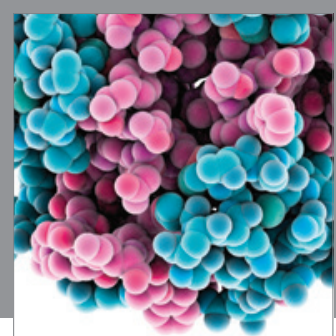

Journal of
Diabetes Research

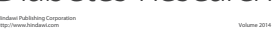

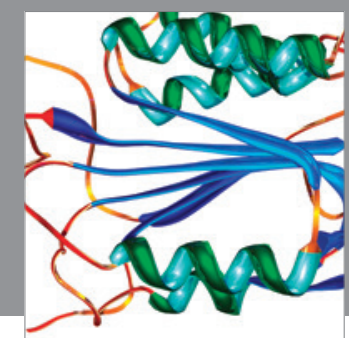

Disease Markers
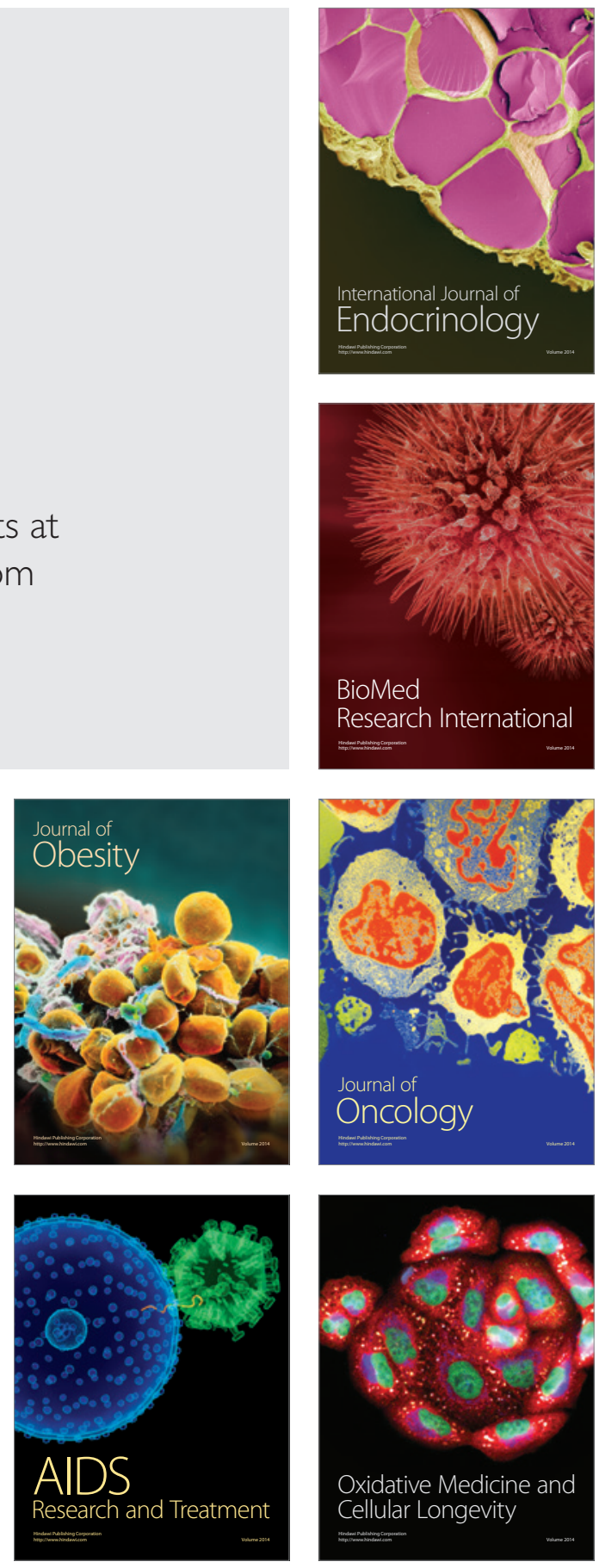